\title{
On the implementation of the eigenvalue method for limit cycle determination in nonlinear systems
}

\author{
KARL HEINZ KIENITZ \\ Institute for Robotics and Mechatronics \\ German Aerospace Center \\ Postfach 1116 \\ D-82230 Weßling, Germany \\ Email: kienitz@ieee.org \\ Tel: +49-8153-28 1471 \\ Fax: +49-8153-28 1441
}

Abstract: In many practical systems, limit cycles can be predicted with suitable precision by frequency domain methods using describing functions. Within such an approach, limit cycles can be predicted using the "eigenvalue method" [7]. This contribution presents a novel and advantageous implementation of this method, using singular value instead of eigenvalue calculations, and enhancing computational efficiency by avoiding a so called “frequency iteration”.

Keywords: describing functions, eigenvalues, limit cycles, Lyapunov equation, nonlinear systems, singular values, stability.

\section{Introduction}

When a dynamic system acts as a low pass filter, limit cycles can be predicted by frequency domain methods in combination with describing functions. Describing functions have been widely discussed throughout the literature, e.g. [1] and [3]. It was shown in [7] that, within such an approach, estimates for frequency and amplitude of limit cycles of a nonlinear system can be determined via eigenvalue calculation. The method was called the "eigenvalue method”. For this purpose a quasi-linear system is derived from the original nonlinear one and the eigenvalue approach is then used. The method is practicable if a model is available consisting of linear differential equations with one or more separable nonlinearities, which may be static or multivalued and which are connected by linear frequency response relations. The approach is not limited to low order systems. Using efficient numerical software for eigenvalue calculation, the method represents a systematic, fast and precise tool [7], provided the low pass filter hypothesis is true. This hypothesis is standard in connection with the application of describing 
functions [1], [3].

This contribution presents a novel and advantageous implementation of the "eigenvalue method" which proposes the use of singular value instead of eigenvalue calculations, and enhances computational efficiency by avoiding a so called "frequency iteration".

\section{A short review of the eigenvalue method}

This section strongly follows [7]. Consider a zero input nonlinear system with separable nonlinearities. A simple example with one nonlinearity is shown in Figure 1. Replace every nonlinear block by a complex gain defined by its sinusoidal describing function [3]. Call this the quasi-linearized system. The sinusoidal describing function presupposes the existence of a sinusoidal oscillation of amplitude $A_{i} \geq 0$ at the entrance of the i-th nonlinear block. The describing function usually is a function of oscillation amplitude, $A_{i}$, at block entrance and in some cases also of oscillation frequency, $\omega$ [3]. It is assumed that, whenever persistent oscillation exists, its frequency is the same throughout the whole system.

If the system has several nonlinear blocks which cannot be lumped into one (i.e. if the nonlinear blocks are neither in series nor in parallel), then at the entrance of every nonlinear block there will be a different amplitude $A_{i}$ (see [3]). System structure will be restricted by the hypothesis that all amplitudes $A_{i}$ of the nonlinear block inputs can be related to one another by functions of frequency, i.e. for any two inputs $A_{j}$ and $A_{k}$ there exists a function of frequency $L_{j k}(\omega)$ such that:

$$
A_{k}=L_{j k}(\omega) A_{j} .
$$

Thus one chooses reference amplitude $A$ and relates all other amplitudes to that one.

As shown in [7], within the validity of the low pass filter hypothesis, system stability can be checked by inspecting the eigenvalues of the (complex) system matrix $F_{N}(A, \omega)$ of the quasi-linearized system:

- all eigenvalues of $F_{N}(A, \omega)$ in the left half of the complex plane for all values of $A$ and $\omega$ indicate system stability;

- $\quad$ an imaginary eigenvalue of $F_{N}(A, \omega)$ with magnitude $\omega$ for some amplitude value $A=A_{L C}$ indicates a limit cycle with amplitude $A_{L C}$ and frequency $\omega$.

The eigenvalue method reduces limit cycle determination to calculation and verification of eigenvalues of $F_{N}(A, \omega)$, while sweeping through the values of $A$ and $\omega$. Because $\omega$ is the absolute value of an eigenvalue 
of $F_{N}$ and at the same time may be an argument of $F_{N}(A, \omega)$, a special procedure called "frequency iteration" was proposed in [7] to calculate $\omega$ in such cases.

As discussed in [7], limit cycle stability can be decided by observing the evolution of the eigenvalues of $F_{N}(A, \omega)$ while applying small variations to limit cycle amplitude $A_{L C}$. Decision criteria are as follows:

a) A limit cycle exists with amplitude $A_{L C}$ and frequency $\omega$ if there exists an imaginary eigenvalue of $F_{N}\left(A_{L C}, \omega\right)$ with magnitude $\omega$.

b) The limit cycle with amplitude $A_{L C}$ and frequency $\omega$ will be stable if:

- $\quad$ all eigenvalues of $F_{N}(A, \omega)$ lie in the left half complex plane for $A=A_{L C}+\delta$, where $\delta$ is a small positive perturbation, and

- $\quad$ some eigenvalue(s) of $F_{N}(A, \omega)$ lie(s) in the right half complex plane for $A=A_{L C}-\delta$.

c) The limit cycle with amplitude $A_{L C}$ and frequency $\omega$ will be unstable if:

- $\quad$ all eigenvalues of $F_{N}(A, \omega)$ lie in the left half complex plane for $A=A_{L C}-\delta$, and

- $\quad$ some eigenvalue(s) of $F_{N}(A, \omega)$ lie(s) in the right half complex plane for $A=A_{L C}+\delta$.

d) The limit cycle with amplitude $A_{L C}$ and frequency $\omega$ will be semi-stable (stable for small, unstable for large $A$ ) if some eigenvalue(s) of $F_{N}(A, \omega)$ lie(s) in the right half complex plane for both, $A=A_{L C}+\delta$ and $A=A_{L C}-\delta$.

e) The limit cycle with amplitude $A_{L C}$ and frequency $\omega$ will be semi-stable (unstable for small, stable at large $A$ ) if all eigenvalues of $F_{N}(A, \omega)$ lie in the left half complex plane for both, $A=A_{L C}+\delta$ and $A=A_{L C}-\delta$.

\section{A novel implementation without eigenvalue calculation}

\subsection{Looking for a limit cycle:}

There exists an imaginary eigenvalue of $F_{N}\left(A_{L C}, \omega\right)$ with magnitude $\omega_{L C}$ iff $\left[j \omega I-F_{N}\left(A_{L C}, \omega\right)\right]$ is singular at $\omega=\omega_{L C}$ [4]. Thus instead of calculating the eigenvalues of $F(A, \omega)$ while sweeping through the values 
of $A$ and $\omega$ in the search for limit cycles, one may look for zero singular values of $[j \omega I-F(A, \omega)]$. This can be conveniently accomplished by solving the minimization problem:

$$
\min _{A, \omega} \underline{\sigma}\left[j \omega I-F_{N}(A, \omega)\right]
$$

where $\underline{\sigma}[$.$] indicates the least singular value. An imaginary eigenvalue of F_{N}\left(A_{L C}, \omega_{L C}\right)$ with magnitude $\omega_{L C}$ will exist iff $\underline{\sigma}\left[j \omega_{L C} I-F_{N}\left(A_{L C}, \omega_{L C}\right)\right]=0$.

This approach has 2 advantages:

- smallest singular value calculation is used instead of calculating all eigenvalues;

- limit cycle frequency is the result of optimization problem (1), rather than the absolute value of an eigenvalue and an optimization parameter. Thus the special procedure of [7] called "frequency iteration" is not needed.

Alternatively, if the system is a unity negative feedback system and the open loop state space representation $\{G(A, \omega), B(A, \omega), C(A, \omega), D(A, \omega)\}$ of the quasi-linearized system is available, one may also solve the following alternative optimization problem without determining the closed loop system matrix:

$$
\min _{A, \omega} \underline{\sigma}\left[\begin{array}{cc}
G(A, \omega)-j \omega I & B(A, \omega) \\
C(A, \omega) & I+D(A, \omega)
\end{array}\right]
$$

An imaginary eigenvalue of $F_{N}\left(A_{L C}, \omega\right)$ with magnitude $\omega_{L C}$ will exist iff

$$
\underline{\sigma}\left[\begin{array}{cc}
G\left(A_{L C}, \omega_{L C}\right)-j \omega_{L C} I & B\left(A_{L C}, \omega_{L C}\right) \\
C\left(A_{L C}, \omega_{L C}\right) & I+D\left(A_{L C}, \omega_{L C}\right)
\end{array}\right]=0
$$

Problems (1) and (2) are equivalent, because looking for imaginary transmission zeros of $\left[\mathrm{I}+C(s I-G)^{-1} B+D\right]$ is equivalent to checking for imaginary closed loop eigenvalues [2], i.e. the eigenvalues of $F_{N}=\left[G-B(I+D)^{-1} C\right]$.

\subsection{Checking the stability of a limit cycle:}

Once a limit cycle is found, the eigenvalue criteria given in section 2 can be used to decide if the limit cycle is stable, unstable or semi-stable. Thus in the original proposal [7] the eigenvalues of $F_{N}(A, \omega)$ are 
tested for $\omega=\omega_{L C}$ and for values of $A$ slightly larger and slightly smaller than $A_{L C}$. Alternatively, the Lyapunov equation $F_{N}{ }^{H} P+P F_{N}=-I$ can be solved for these situations and then the positive definiteness of solution $P$ (which is a necessary and sufficient stability condition) checked by one of the following criteria:

- A positive definite Hermitian matrix has positive principal minors.

- A positive definite Hermitian matrix has only positive eigenvalues.

- The Cholesky decomposition exists for positive definite Hermitian matrices.

Higham [5] suggests the use of a Cholesky factorization attempt as a stable and efficient test for positive definiteness. Such use of Cholesky factorization algorithms is supported by numerical tools currently in use (such as Matlab $^{\circledR}$ ) and therefore is suggested here as an alternative test for limit cycle stability verification through Lyapunov equation solution. Henceforth this test will be called the Lyapunov Cholesky stability test.

\section{Examples}

To illustrate the application of the proposed implementation and its advantage, two systems will be considered of the general form given in Figure 1.

Let the linear dynamics be:

$$
\begin{aligned}
& \dot{x}=G x+B u \\
& y=C x
\end{aligned}
$$

with

$$
G=\left[\begin{array}{cccc}
-1 & 1 & 0 & 0 \\
0 & -2 & 0 & 0 \\
1 & 0 & -10 & 0 \\
0 & 0 & 1 & 0
\end{array}\right], B=\left[\begin{array}{l}
0 \\
2 \\
0 \\
0
\end{array}\right], C=\left[\begin{array}{llll}
0 & 0 & 0 & 10
\end{array}\right]
$$

Two different nonlinearities with describing functions $N_{1}(A)$ and $N_{2}(A, \omega)$ will be considered, where $A$ is oscillation amplitude at the nonlinearity's input and $\omega$ is the oscillation frequency in rd/s. Let the describing functions be: 


$$
\begin{gathered}
N_{1}(A)=\frac{4}{A} \sqrt{1-\left(\frac{1}{A}\right)^{2}}-j\left(\frac{2}{A}\right)^{2} \\
N_{2}(A, \omega)=\frac{4}{A} e^{-j \frac{\omega}{4}} \cos \left(\frac{\omega}{4}\right)
\end{gathered}
$$

$N_{1}(A)$ is the describing function of a relay of amplitude $\pi$ and appropriate hysteresis width [3]. $N_{2}(A, \omega)$ is the describing function of a switching actuator with amplitude $\pi$, subject to switching restrictions as described in [6].

For these two systems, the closed loop approach (Equation 1) and the open loop approach (Equation 2) were applied. Results were compared to those obtained with an implementation of the original eigenvalue method proposal [7]. In all cases, values obtained for $A_{L C}$ and $\omega_{L C}$ were equal up to five significant digits.

For System 1, a system described by Figure 1 and Equations 3, 4 and 5, calculations yield a stable limit cycle with amplitude $A_{L C}=3.0467$ and $\omega_{L C}=0.89152 \mathrm{rd} / \mathrm{s}$. For System 2, a system described by Figure 1 and Equations 3, 4 and 6, calculations yield a stable limit cycle with amplitude $A_{L C}=2.5376$ and $\omega_{L C}=$ $0.97779 \mathrm{rd} / \mathrm{s}$. Within the usual error bounds known for the describing function method, these values match simulation results.

Computational costs were assessed using flop counts in Matlab ${ }^{\circledR}$. Table 1 summarizes the results. Procedures 1 to 3 are procedures without search. Flop counts for procedures 4 - 9 depend on the search methods used, as well as on single iteration costs, which are those of procedures 1 - 3. Procedures 4, 5, 7 and 8 used the simplex search from Matlab ${ }^{\circledR}$ Optimization Toolbox. Procedures 6 and 9 used scalar bounded search from the same toolbox.

From Table 1 it is seen that the implementation proposed herein yields expressive gains over the original proposal from [7] when the so-called "frequency iteration" is needed, i.e. when $F_{N}$ is a function of $A$ and $\omega$, as is the case of System 2. When this is not the case, as for System 1, the original proposal results in less computational cost, because the determination of $A_{L C}$ and $\omega_{L C}$ is done sequentially.

The Lyapunov - Cholesky limit cycle stability turns out to be approximately 3 to 5 times more costly than the direct eigenvalue verification outlined in section 2. The Lyapunov - Cholesky stability test for the limit cycle of System 1 resulted in a flop count of 26,552 against 7,978 for the direct eigenvalue test. For 
the limit cycle of System 2 the flop counts were 27,642 and 8,932 respectively. Thus, generally the direct eigenvalue test is to be preferred. However, the Lyapunov - Cholesky test stands as a viable alternative verification tool.

\section{Comments and conclusion}

In the foregoing sections a novel implementation of the eigenvalue method for limit cycle determination of nonlinear systems was proposed. The main advantage over the original proposal of the method in [7] is the avoidance of the so called „frequency iteration“. Additionally, singular value calculations generically are more robust and less computationally expensive than eigenvalue calculations. Thus the proposed implementation results in significantly less computational effort in all cases were in the original proposal a „frequency iteration“ is needed.

Limit cycle stability can be checked via eigenvalue verification, as in the original proposal, or alternatively via a Lyapunov - Cholesky test outlined in section 3.2. Experiments with built-in implementations of eigenvalue calculation and Lyapunov equation solution in Matlab ${ }^{\circledR}$ indicate that the approach to limit cycle stability check via eigenvalue calculation is computationally less expensive.

\section{Acknowledgements}

The author is on leave from Instituto Tecnológico de Aeronáutica, Brazil, and acknowledges partial support by the Alexander von Humboldt Foundation, Germany, and Fundação de Amparo à Pesquisa do Estado de São Paulo, Brazil.

\section{References}

[1] Cook, P., Nonlinear Dynamical Systems, Prentice-Hall, Englewood Cliffs, N.J., 1986.

[2] Emami-Naeini, A. and Van Dooren, P., 'Computation of zeros of linear multivariable systems', Automatica 18 (4), 1982, 415-430.

[3] Gelb, A. and Velde, W.E.V., Multiple-Input Describing Functions and Nonlinear System Design, McGraw-Hill, New York, 1968. 
[4] Gantmacher, F.R., Matrizenrechnung, VEB Verlag, Berlin, 1958.

[5] Higham, N.J., 'Computing a nearest symmetric positive semidefinite matrix', Linear Algebra and Its Applications 103, 1988, 103-118.

[6] Oliveira, N.M.F. and Kienitz, K.H., 'Attitude controller design for a system using actuators with switching-time restrictions and delays', in Proceedings of the AIAA Guidance, Navigation, and Control Conference, Denver, CO, August 14-17, 2000, Paper AIAA-2000-3967.

[7] Somieski, G., 'An eigenvalue method for calculation of stability and limit cycles in nonlinear systems’, Nonlinear Dynamics 26 (1), 2001, 3-22. 
FIGURE:

Fig. 1: Control system with linear subsystem and separable nonlinearity

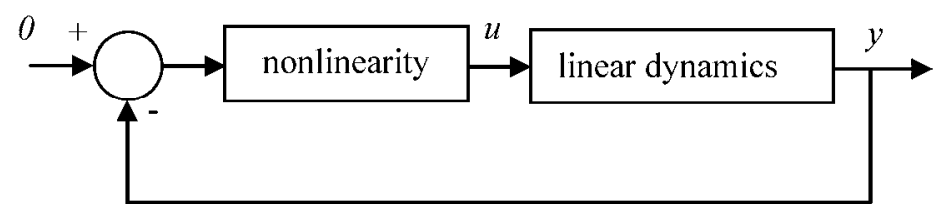




\section{TABLE:}

Table 1: Assessment of computational cost

\begin{tabular}{|c|c|c|c|}
\hline Procedure label & \multicolumn{2}{|l|}{ Procedure description } & FLOP count \\
\hline 1 & \multicolumn{2}{|c|}{$\underline{\sigma}\left[j \omega I-F_{N}(A, \omega)\right]$ for System 1} & 1,626 \\
\hline 2 & $\underline{\sigma}\left[\begin{array}{cc}G(A, \omega)-j \omega I & B(A, \omega) \\
C(A, \omega) & I+D(A, \omega)\end{array}\right]$ & for System 1 & 2,363 \\
\hline 3 & Calculation of the eigenvalues of $F_{N}(A$ & $4, \omega)$ for System 1 & 4,660 \\
\hline 4 & $\min _{A, \omega} \underline{\sigma}\left[j \omega I-F_{N}(A, \omega)\right]$ for $\mathrm{S}$ & ystem 1 & 134,529 \\
\hline 5 & $\min _{A, \omega} \underline{\sigma}\left[\begin{array}{cc}G(A, \omega)-j \omega I & B(A, \omega) \\
C(A, \omega) & I+D(A, \omega)\end{array}\right]$ & for System 1 & 181,490 \\
\hline 6 & $\begin{array}{l}\text { Determination of } A, \omega \text { for System } 1 \text { as } \\
\text { (no "frequency iteration" ne }\end{array}$ & $\begin{array}{l}\text { described in [7] } \\
\text { eeded) }\end{array}$ & 71,337 \\
\hline 7 & $\min _{A, \omega} \underline{\sigma}\left[j \omega I-F_{N}(A, \omega)\right]$ for $\mathrm{S}$ & ystem 2 & 143,584 \\
\hline 8 & $\min _{A, \omega} \underline{\sigma}\left[\begin{array}{cc}G(A, \omega)-j \omega I & B(A, \omega) \\
C(A, \omega) & I+D(A, \omega)\end{array}\right]$ & for System 2 & 200,835 \\
\hline 9 & $\begin{array}{r}\text { Determination of } A, \omega \text { for System } 2 \text { as } \\
\text { ("frequency iteration" nee }\end{array}$ & described in [7] & 339,383 \\
\hline
\end{tabular}

